\title{
PDRK: A General Kinetic Dispersion Relation Solver for Magnetized Plasma
}

\author{
Hua-sheng XIE, Yong Xiao \\ Institute for Fusion Theory and Simulation and the Department of Physics, Zhejiang University, Hangzhou, 310027, People's Republic of China
}

\begin{abstract}
A general, fast, and effective approach is developed for numerical calculation of kinetic plasma dispersion relations. The plasma dispersion function is approximated by $J$-pole expansion. Subsequently, the dispersion relation is transformed to a standard matrix eigenvalue problem of an equivalent linear system. The result is accurate for $J=8$ except the solutions that are the little interesting heavily damped modes. In contrast to conventional approaches, such as Newton's iterative method, this approach can give either all the solutions in the system or a few solutions around the initial guess. It is also free from convergent problems. The approach is demonstrated from electrostatic one-dimensional and three-dimensional dispersion relations, to electromagnetic kinetic magnetized plasma dispersion relation for bi-Maxwellian distribution with parallel velocity drift.
\end{abstract}

\section{Program summary}

\section{Title of program: PDRK}

Catalogue identifier:

Program summary URL:

Program obtainable from: CPC Program Library, Queen University of Belfast, N. Ireland

Computer for which the program is designed and others on which it has been tested: Computers: Any computer running MATLAB 7. Tested on Lenovo T430.

Operating systems under which the program has been tested: Windows 8

Programming language used: MATLAB 7

Memory required to execute with typical data: $500 \mathrm{M}$

No. of lines in distributed program, including test data, etc.: 700

No. of bytes in distributed program, including test data, etc.: 30000

Distribution format: .tar.gz

Nature of physical problem: Solving kinetic dispersion relations for multi-species plasmas.

Method of solution: Transforming to an equivalent linear system and then solving as matrix eigenvalue problem.

Restrictions on the complexity of the problem: Not suitable for heavily damped modes and only non-relativistic version at present.

Typical running time: About 1 minutes on a Intel $2.60 \mathrm{GHz}$ PC.

Unusual features of the program: Can give all interesting solutions fastly and without convergent difficulty.

Keywords: Plasma physics, Dispersion relation, Kinetic, Waves, Instabilities, Linear system, Matrix eigenvalue PACS: 52.27.Cm, 52.35.Qz, 52.35.-g, 52.35.Fp, 52.25.Dg

\section{Introduction}

Given the richness of waves and instabilities in astrophysical, space, laser, and laboratory plasmas, studying the corresponding linear dispersion relations of different plasma systems is of practical interest. However, except for some

Email address: huashengxie@gmail.com (Hua-sheng XIE) 
simple cases, the dispersion relations are usually too complicated to be solved either analytically or even numerically.

The multi-fluid plasma dispersion relation has been numerically solved generally using matrix method in a previous work, i.e., PDRF[1].

At present, several multi-component magnetized kinetic plasma dispersion relations solvers are available, such as WHAMP by Ronnmark[2, 3], NHDS by Verscharen et al. [4], and solvers by Gary et al. [5, 6], by Willes and Cairns [7, 8] and by Lin et al. [9], among others. However, all these solvers obtain the dispersion relations from the determinant of the corresponding 3-by-3 dielectric tensor using a given initial guess. These solvers are usually time consuming and have difficulty showing a complete picture of the modes in the system. Furthermore, these solvers may also suffer from convergence problems because the plasma dispersion function $Z(\zeta)$ and Bessel functions (especially in high-order cyclotron frequencies, e.g., $\omega>10 \Omega_{c}$, where $\Omega_{c}$ is the cyclotron frequency) have several solutions around a given frequency. Thus, a careful selection of the initial guess is required to make it converge to the solution we want.

In this work, we extend our previous work, a multi-fluid dispersion relation solver[1], to a general kinetic version, but still maintain the use of a full-matrix approach. In contrast, two additional steps are required in the kinetic version: solving for the plasma dispersion function $Z(\zeta)$ and seeking an equivalent linear system. The first step is accomplished by $J$-pole expansion (Padé approximation) as used by Martin et al.[10] and Ronnmark[2, 3]. The first step has also been used by Cereceda and Puerta[11] to solve the electrostatic 1D (ES1D) system. Physical interpretations of the Padé approximation of $Z(\zeta)$ are given by Tjulin et al.[12] and Robinson and Newman[13]. The second step is more difficult and should be treated on a case-to-case basis as we can see in the following sections.

\section{Electrostatic systems}

We start with simple electrostatic systems to show how our approach can be implemented.

\subsection{Electrostatic $1 D$}

First, we solve the simplest multi-component electrostatic 1D (ES1D) problem with drift Maxwellian distribution $f_{s 0}=\left(\frac{m_{s}}{2 \pi k_{B} T_{s}}\right)^{1 / 2} \exp \left[-\frac{\left(v-v_{s 0}\right)^{2}}{2 k_{B} T_{s}}\right]$. The dispersion relation is

$$
D=1+\sum_{s=1}^{S} \frac{1}{\left(k \lambda_{D s}\right)^{2}}\left[1+\zeta_{s} Z\left(\zeta_{s}\right)\right]=0
$$

where $\lambda_{D s}^{2}=\frac{\epsilon_{0} k_{B} T_{s}}{n_{s} q_{s}^{2}}, v_{t s}=\sqrt{\frac{2 k_{B} T_{s}}{m_{s}}}$ and $\zeta_{s}=\frac{\omega-k v_{s 0}}{k v_{t s}}$. Unmentioned notations are standard. The plasma dispersion function can be approximated using $J$-pole expansion

$$
Z(\zeta) \simeq Z_{J}(\zeta)=\sum_{j=1}^{J} \frac{b_{j}}{\zeta-c_{j}},
$$

where $J=8$ is used by Ronnmark [2, 3] and $J=2,3,4$ are provided by Martin et al. [10], producing accurate results for most domains (except $y<\sqrt{\pi} x^{2} e^{-x^{2}}$ when $x \gg 1$, with $\zeta=x+i y$ ), especially in the upper plane. However, the method does not perform well for heavily damped modes, which are of little interest anyway. For completeness, the coefficients $c_{j}$ and $b_{j}$ for $J=4, J=8$ and $J=12$ (see Appendix A) are provided in Table 1 Note the useful relations $\sum_{j} b_{j}=-1, \sum_{j} b_{j} c_{j}=0$ and $\sum_{j} b_{j} c_{j}^{2}=-1 / 2$.

Combining (1) and 2, yields

$$
1+\sum_{s} \sum_{j} \frac{b_{s j}}{\left(\omega-c_{s j}\right)}=0
$$

with $b_{s j}=\frac{b_{j} c_{j} v_{t s}}{k \lambda_{D s}^{2}}$ and $c_{s j}=k\left(v_{s 0}+v_{t s} c_{j}\right)$. An equivalent linear system can be obtained as follows:

$$
\begin{gathered}
\omega n_{s j}=c_{s j} n_{s j}+b_{s j} E, \\
E=-\sum_{s j} n_{s j},
\end{gathered}
$$




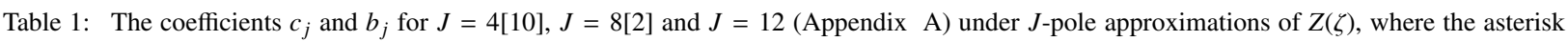
denotes complex conjugation.

\begin{tabular}{ccc}
\hline \hline \multirow{3}{*}{$=4$} & $b_{1}=0.546796859834032+0.037196505239277 \mathrm{i}$ & $c_{1}=1.23588765343592-1.21498213255731 \mathrm{i}$ \\
& $b_{2}=-1.046796859834027+2.101852568038518 \mathrm{i}$ & $c_{2}=-0.378611612386277-1.350943585432730 \mathrm{i}$ \\
& $b(3: 4)=b^{*}(1: 2)$ & $c(3: 4)=-c^{*}(1: 2)$ \\
\hline \multirow{3}{*}{$=8$} & $b_{1}=-1.734012457471826 \mathrm{E}-2-4.630639291680322 \mathrm{E}-2 \mathrm{i}$ & $c_{1}=2.237687789201900-1.625940856173727 \mathrm{i}$ \\
& $b_{2}=-7.399169923225014 \mathrm{E}-1+8.395179978099844 \mathrm{E}-1 \mathrm{i}$ & $c_{2}=1.465234126106004-1.789620129162444 \mathrm{i}$ \\
& $b_{3}=5.840628642184073+9.536009057643667 \mathrm{E}-1 \mathrm{i}$ & $c_{3}=0.8392539817232638-1.891995045765206 \mathrm{i}$ \\
& $b_{4}=-5.583371525286853-1.120854319126599 \mathrm{E} 1 \mathrm{i}$ & $c_{4}=0.2739362226285564-1.941786875844713 \mathrm{i}$ \\
& $b(5: 8)=b^{*}(1: 4)$ & $c(5: 8)=-c^{*}(1: 4)$ \\
\hline \multirow{4}{*}{$b_{1}=-0.004547861216840+0.000621096229879 \mathrm{i}$} & $c_{1}=2.978429162453205-2.049696666440972 \mathrm{i}$ \\
$b_{2}=0.215155729059403-0.201505401705763 \mathrm{i}$ & $c_{2}=-2.256783783969929-2.208618411911446 \mathrm{i}$ \\
$b_{3}=0.439545043457674-4.161084685092405 \mathrm{i}$ & $c_{3}=1.673799856114519-2.324085194217706 \mathrm{i}$ \\
& $b_{4}=-20.216967308177410+12.885503528244977 \mathrm{i}$ & $c_{4}=1.159032034062764-2.406739409567887 \mathrm{i}$ \\
$b_{5}=67.081488119986460-20.846345891864550 \mathrm{i}$ & $c_{5}=-0.682287637027822-2.460365014999888 \mathrm{i}$ \\
& $b_{6}=-4.801467372237129 \mathrm{e}+01-1.072756140299431 \mathrm{e}+02 \mathrm{i}$ & $c_{6}=0.225365375295874-2.486779417872603 \mathrm{i}$ \\
& $b(7: 12)=b^{*}(1: 6)$ & $c(7: 12)=-c^{*}(1: 6)$ \\
\hline
\end{tabular}

Table 2: Comparison of the Landau damping solutions using the matrix method and the original $Z(\zeta)$ function. Here, $\omega$ is normalized by $\omega_{p e}=\sqrt{n_{e} e^{2} / \epsilon_{0} m_{e}}$.

\begin{tabular}{ccccccccc}
\hline$k \lambda_{D e}$ & $\omega_{r}^{M}(J=4)$ & $\omega_{i}^{M}(J=4)$ & $\omega_{r}^{M}(J=8)$ & $\omega_{i}^{M}(J=8)$ & $\omega_{r}^{M}(J=12)$ & $\omega_{i}^{M}(J=12)$ & $\omega_{r}^{Z}$ & $\omega_{i}^{Z}$ \\
\hline 0.1 & 0.9956 & $9.5 \mathrm{E}-3$ & 1.0152 & $1.7 \mathrm{E}-5$ & 1.0152 & $9.5 \mathrm{E}-8$ & 1.0152 & $-4.8 \mathrm{E}-15$ \\
0.5 & 1.4235 & -0.1699 & 1.4156 & -0.1534 & 1.4157 & -0.1534 & 1.4157 & -0.1534 \\
1.0 & 2.0170 & -0.8439 & 2.0459 & -0.8514 & 2.0458 & -0.8513 & 2.0458 & -0.8513 \\
2.0 & 3.2948 & -2.6741 & 3.1893 & -2.8272 & 3.1891 & -2.8272 & 3.1891 & -2.8272 \\
\hline \hline
\end{tabular}

which is an eigenvalue problem of a $S J \times S J$ dimensional eigen matrix $\boldsymbol{M}$, i.e., $\omega X=\boldsymbol{M} \boldsymbol{X}$, with $S J=S \times J$ and $\boldsymbol{X}=\left\{n_{s j}\right\}$. The singularity in the denominator of $(3$, which is encountered in conventional methods, can be canceled by using the transformation (4). Hence, the matrix method can easily support multi-component systems.

For Langmuir wave Landau damping, calculating the largest imaginary part solution using matrix method $\left(\omega^{M}\right)$ and the original $Z(\zeta)$ function $\left(\omega^{Z}\right)[14]$ are shown in Table 2 We can see that the result of the matrix method is accurate in $10^{-4}$ when $J=8$ and the error for $J=4$ is also small (10\%). Thus, we have verified that our approach is feasible. In principle, infinite numbers of frequency solutions exist for a fixed wave vector $k$ (the physical discussions can be found in Ref.[15] and references in). Fig 1 shows all the solutions of the matrix method and the solutions using $Z(\zeta)$ function for $k \lambda_{D e}=0.8$. The largest imaginary part solutions (first solution) are almost identical, which is our objective. However, other heavily damped solutions should be excluded due to the poor approximation in those ranges. For example, the error for the second solution between the $Z(\zeta)$ solution and the $J=8$ solution is around $10 \%$, whereas the third solution is completely wrong for $J=8$. Fortunately, for most studies, these heavily damped modes are of little interest. The $J=12$ results can be more accurate $\left(10^{-7}\right)$ as shown in Table 2 and Fig 1 In principle, Eq. (1) has no singularity for $k \neq 0$. Given the existence of multiple solutions, if the initial guess is not good, then root finding cannot converge to the desired solutions.

For the two-frequency-scale ion acoustic mode, besides the Langmuir mode $\omega=2.0459-0.8513 i$, the largest imaginary part solution obtained from the matrix method $(J=8)$ is also consistent with the solution obtained from the $Z(\zeta)$ function, e.g., $T_{i}=T_{e}, m_{i}=1836 m_{e}, k \lambda_{D e}=1$, gives $\omega=0.0420-0.0269 i$. Hereafter, $J=8$ will be used as default.

We further check the electron bump-on-tail mode $(s=e, b)$, with $T_{b}=T_{e}, v_{b}=5 v_{t e}$ and $n_{b}=0.1 n_{0}\left(n_{e}=n_{0}-n_{b}\right)$. Both $J=8$ matrix method and root finding using $Z(\zeta)$ function give the same largest imaginary part solution $\omega=$ 


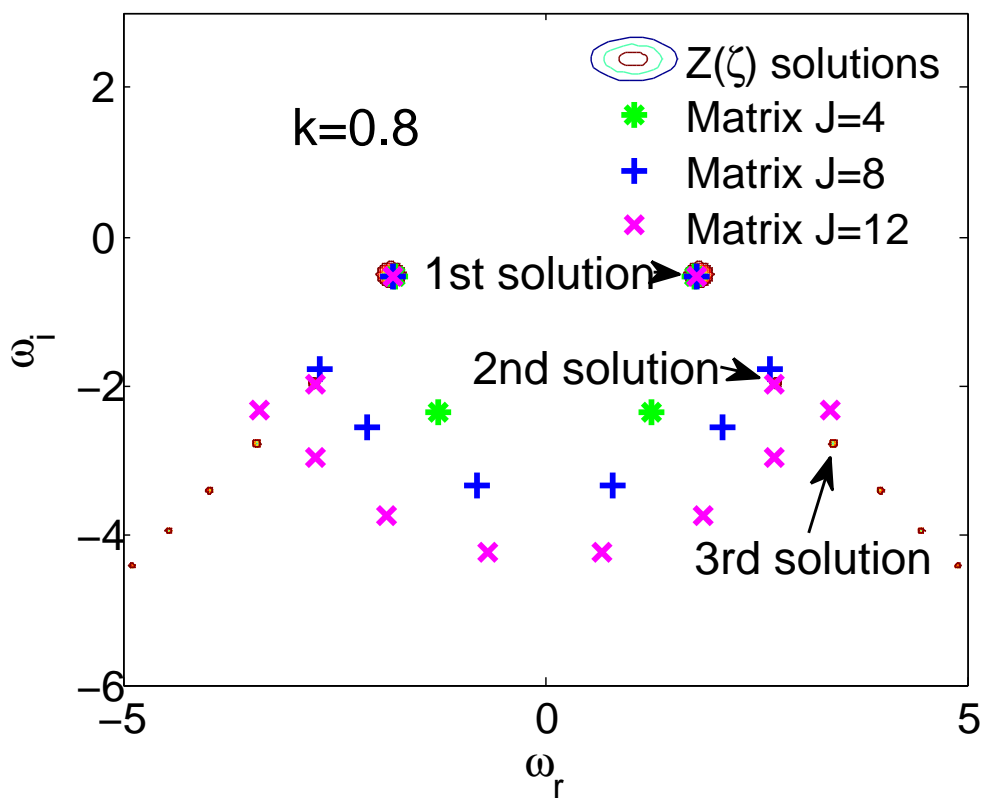

Figure 1: Comparison of all the solutions obtained from the matrix method and the $Z(\zeta)$ function.
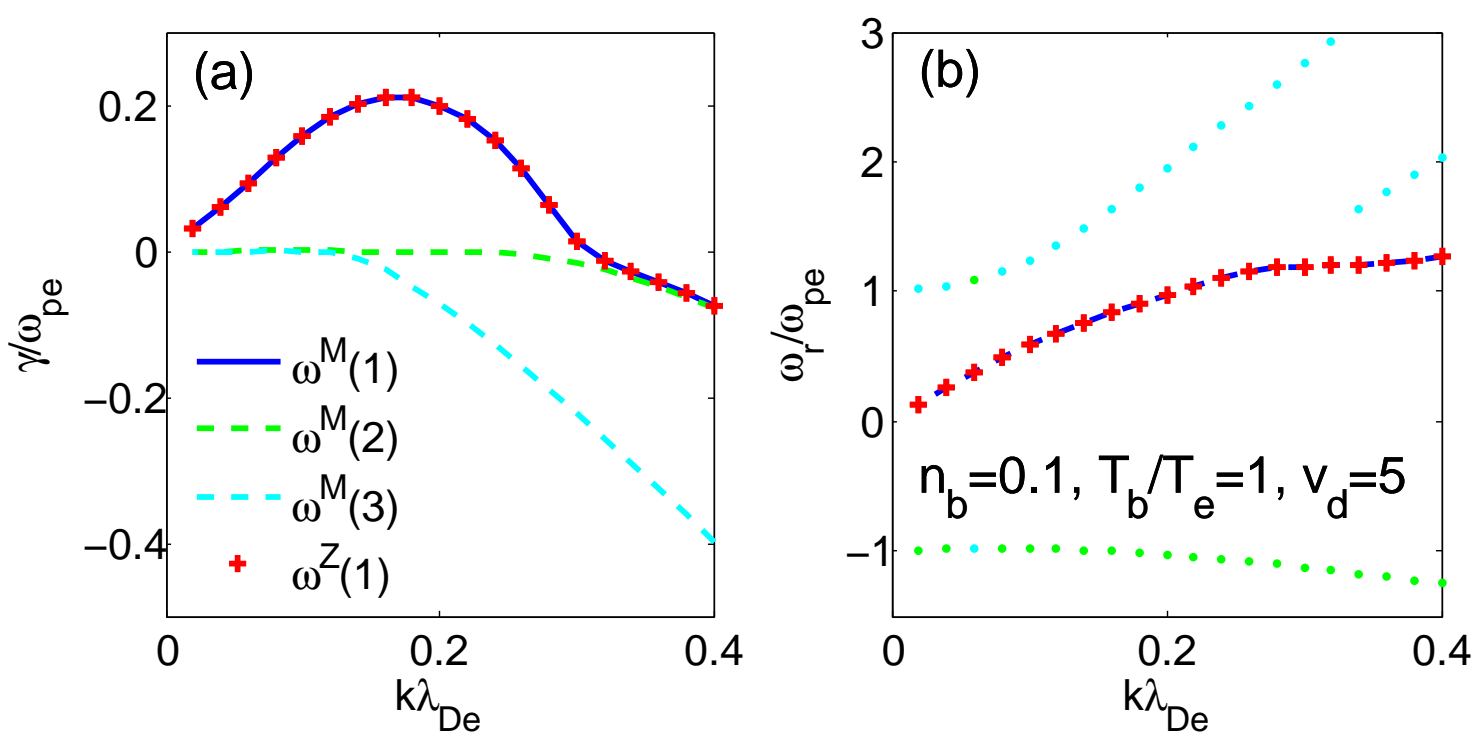

Figure 2: Comparison of the first three $\left(\omega^{M}\right)$ largest imaginary part solutions obtained from the matrix method $(J=8)$ and one solution $\left(\omega^{Z}\right)$ obtained from $Z(\zeta)$ function for the bump-on-tail parameters. 
$0.9785+0.2000 i$ for $k \lambda_{D e}=0.2$. The $J=4$ matrix method gives $\omega=0.9772+0.2076 i$. Fig 2 shows $\omega$ and $\gamma$ vs. $k$ for the above parameters, where the first three largest imaginary part solutions from the matrix method $(J=8)$ and one solution from $Z(\zeta)$ function are shown. $\omega^{Z}$ is identical to $\omega^{M}$. However, different initial guesses should be tested to find other solutions when we using the $Z(\zeta)$ function. By contrast, no initial guess is required when using the matrix method. Therefore, with matrix method, no important solutions are missed.

\subsection{Harris dispersion relation}

We go further to solve a more complicated example, including the $n$-th ( $n=-\infty$ to $\infty$ ) order cyclotron frequency, i.e., the electrostatic 3D-magnetized (ES3D) Harris dispersion relation[16]

$$
D=1+\sum_{s=1}^{S} \frac{1}{\left(k \lambda_{D s}\right)^{2}}\left[1+\frac{\omega-k_{z} v_{s 0}-n \Omega_{s}+\lambda_{T} n \Omega_{s}}{k_{z} v_{z t s}} \sum_{n=-\infty}^{\infty} \Gamma_{n}\left(b_{s}\right) Z\left(\zeta_{s n}\right)\right]=0
$$

where, $\lambda_{D s}^{2}=\frac{\epsilon_{0} k_{B} T_{z s}}{n_{s 0} q_{s}^{2}}, v_{t s}=\sqrt{\frac{2 k_{B} T_{s}}{m_{s}}}, \lambda_{T}=T_{z} / T_{\perp}, \zeta_{s n}=\frac{\omega-k_{z} v_{s 0}-n \Omega_{s}}{k_{z} v_{z t s}}, \Gamma_{n}(b)=I_{n}(b) e^{-b}, b_{s}=k_{\perp}^{2} \rho_{c s}^{2}, \rho_{c s}=\sqrt{\frac{v_{\perp t s}^{2}}{\Omega_{s}}}, I_{n}$ is the modified Bessel function, and the equilibrium distribution is assumed to be drift bi-Maxwellian $f_{s 0}=f_{\perp}\left(v_{\perp}\right) f_{z}\left(v_{z}\right)$, with $f_{\perp}=\frac{m_{s}}{2 \pi k_{B} T_{s \perp}} \exp \left[-\frac{m_{s} v_{\perp}^{2}}{2 k_{B} T_{s \perp}}\right]$ and $f_{z}=\left(\frac{m_{s}}{2 \pi k_{B} T_{s \perp}}\right)^{1 / 2} \exp \left[-\frac{m_{s}\left(v_{\|}-v_{s 0}\right)^{2}}{2 k_{B} T_{s z}}\right]$. The background magnetic field is assumed to be $\boldsymbol{B}_{0}=\left(0,0, B_{0}\right)$, and the wave vector $\boldsymbol{k}=\left(k_{x}, 0, k_{z}\right)=(k \sin \theta, 0, k \cos \theta)$, which gives $k_{\perp}=k_{x}$ and $k_{\|}=k_{z}$.

This dispersion relation contains infinite-order summation of Bessel functions. However, Eq. (5) is very similar to Eq.(1). Thus, the transformation to an equivalent linear system/matrix is the same and straightforward. In the computation, we only keep the first $N$ Bessel functions, i.e., $n=-N$ to $N$. The dimensions of the eigen matrix is $S N J \times S N J$, with $S N J=S \times(2 N+1) \times J$. The singularity for $k_{z} \rightarrow 0$ around $\omega-n \Omega_{c s} \rightarrow 0$ in $(5)$ is removed after the transformation.

\subsubsection{Electron Bernstein modes}

First, we benchmark the electron Bernstein modes $(s=e)$. The result is shown in Fig 3 (a), with parameter $\omega_{p e}=2.5 \omega_{c e}$. For the modes with frequency $\omega<6 \omega_{c}$, considering only the $N=10$-order Bessel functions is accurate enough. The upper hybrid frequency calculated at the cold limit is $\omega_{U H}=\sqrt{\omega_{c}^{2}+\omega_{p}^{2}}=2.69$, which is consistent with the matrix solution in the limit $k_{\perp} \rho_{c} \rightarrow 0$. Fig 3 (a) also agrees with Fig.9.8 in Ref.[16]. The corresponding ES1D3V particle-in-cell (PIC) simulation (ion immobile, $k=k_{\perp}$ ) verification is also shown in Fig $3(\mathrm{~b})$, where good agreement is observed.

\subsubsection{Anisotropic instabilities}

Second, we benchmark the anisotropic instabilities with Ref.[17]. The contour plot of the growth rate $\gamma / \omega_{c}$ is shown in Fig 4 , with $\omega_{p}=\omega_{c}$ and $N=4$. The results agree with Fig.2 in Ref.[17].

\section{Electromagnetic dispersion relation}

In the above section, we have shown that the matrix method can solve the kinetic dispersion relations. In addition, the results are accurate enough even if we used Padé approximation to the $Z$ function, which gives us enough confidence with the approach to extend its application further to the magnetized electromagnetic (EM3D) dispersion relations, which has not been solved well using conventional approaches.

\subsection{The dispersion relation}

The equilibrium distribution is still assumed to be drift bi-Maxwellian as in Sec 2.2 and also $\boldsymbol{B}_{0}=\left(0,0, B_{0}\right)$ and $\boldsymbol{k}=\left(k_{x}, 0, k_{z}\right)$. The dispersion relation can be derived as [18]

$$
\left|\begin{array}{ccc}
K_{x x}-\frac{c^{2} k^{2}}{\omega^{2}} \cos ^{2} \theta & K_{x y} & K_{x z}+\frac{c^{2} k^{2}}{\omega^{2}} \sin \theta \cos \theta \\
K_{y x} & K_{y y}-\frac{c^{2} k^{2}}{\omega^{2}} & K_{y z} \\
K_{z x}+\frac{c^{2} k^{2}}{\omega^{2}} \sin \theta \cos \theta & K_{z y} & K_{z z}-\frac{c^{2} k^{2}}{\omega^{2}} \sin ^{2} \theta
\end{array}\right|=0,
$$


(a) PDRK solutions, $\omega_{\mathrm{p}} / \omega_{\mathrm{c}}=2.5, \mathrm{~N}=10$

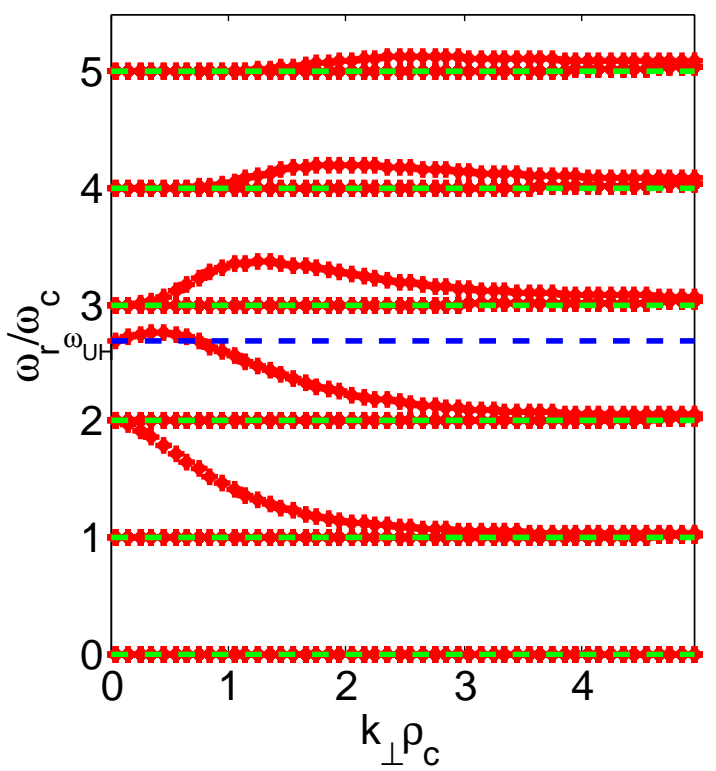

(b) PIC spectral vs. PDRK

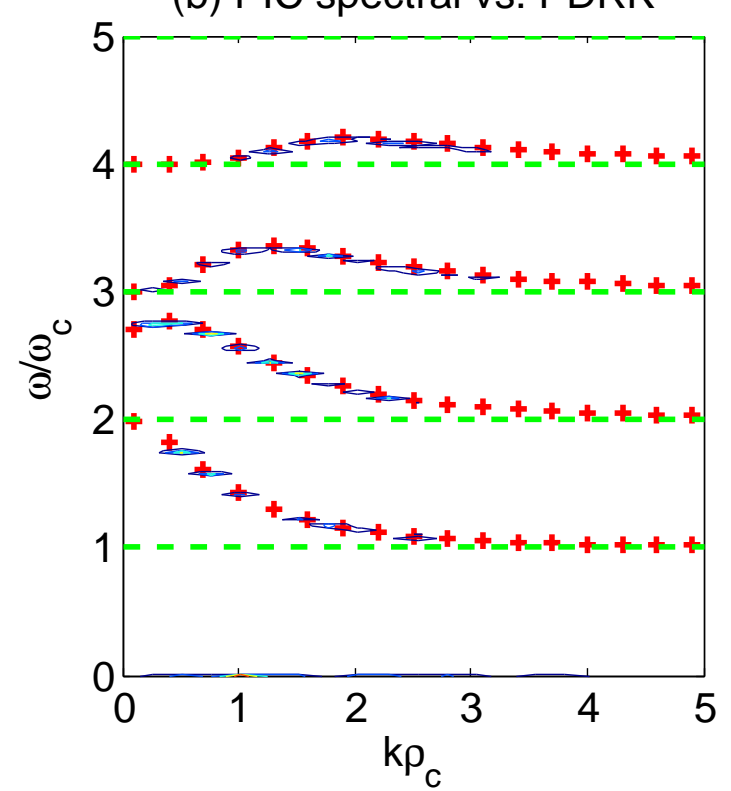

Figure 3: The electron Bernstein modes calculated from the Harris dispersion relation using the matrix method. The upper hybrid frequency calculated at the cold limit is $\omega_{U H}=\sqrt{\omega_{c}^{2}+\omega_{p}^{2}}=2.69$, which agrees with the matrix solution in the limit $k_{\perp} \rho_{c} \rightarrow 0$. The plasma dispersion relation kinetic version (PDRK-ES3D) solutions also agree with the contour plot of the PIC spectra (b).
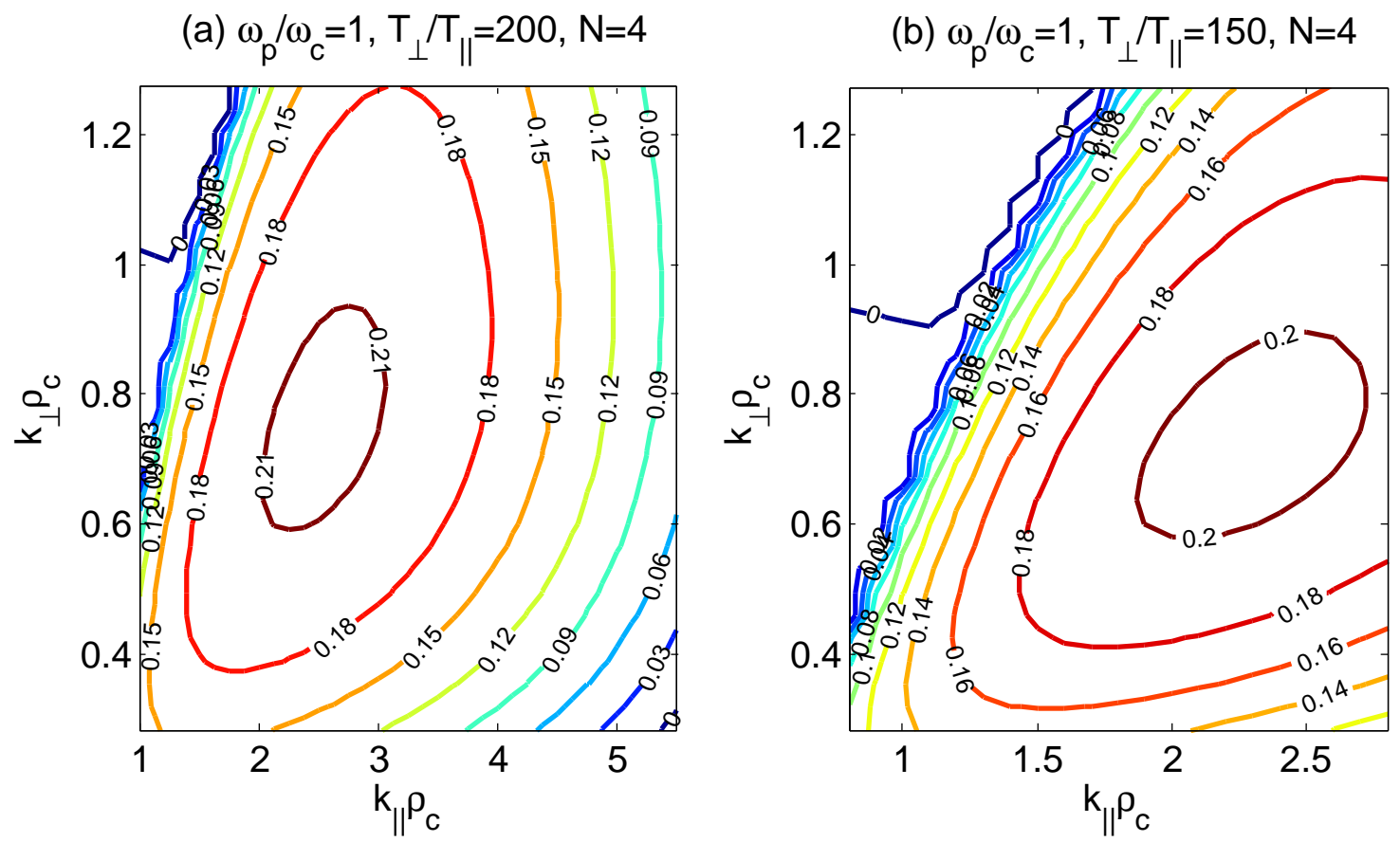

Figure 4: The anisotropic instabilities (growth rate $\gamma / \omega_{c}$ ) calculated from the Harris dispersion relation using the matrix method. The results agree with Ref.[17]. 
with $\boldsymbol{K}=\boldsymbol{I}+\sum_{s} \frac{\omega_{p s}^{2}}{\omega^{2}}\left[\sum_{n}\left\{\zeta_{0} Z\left(\zeta_{n}\right)-\left(1-\frac{1}{\lambda_{T}}\right)\left[1+\zeta_{n} Z\left(\zeta_{n}\right)\right\} \boldsymbol{X}_{n}+2 \eta_{0}^{2} \lambda_{T} \boldsymbol{L}\right]\right.$, where

$$
\boldsymbol{X}_{n}=\left(\begin{array}{ccc}
n^{2} \Gamma_{n} / b & i n \Gamma_{n}^{\prime} & -\left(2 \lambda_{T}\right)^{1 / 2} \eta_{n} \frac{n}{\alpha} \Gamma_{n} \\
i n \Gamma_{n}^{\prime} & n^{2} / b \Gamma_{n}-2 b \Gamma_{n}^{\prime} & i\left(2 \lambda_{T}\right)^{1 / 2} \eta_{n} \alpha \Gamma_{n}^{\prime} \\
-\left(2 \lambda_{T}\right)^{1 / 2} \eta_{n} \frac{n}{\alpha} \Gamma_{n} & -i\left(2 \lambda_{T}\right)^{1 / 2} \eta_{n} \alpha \Gamma_{n}^{\prime} & 2 \lambda_{T} \eta_{n}^{2} \Gamma_{n}
\end{array}\right),
$$

$\eta_{n}=\frac{\omega+n \Omega}{k_{v} T_{z}}, \lambda_{T}=\frac{T_{z}}{T_{\perp}}, b=\left(\frac{k_{k} v_{T \perp}}{\Omega}\right)^{2}, \alpha=\frac{k_{k} v_{T \perp}}{\Omega}, v_{T_{z}}^{2}=\frac{k_{B} T_{z}}{m}, v_{T_{\perp}}^{2}=\frac{k_{B} T_{\perp}}{m}$ and the matrix components of $\boldsymbol{L}$ are all zero, except for $L_{z z}=1$.

\subsection{The linear transformation}

To seek an equivalent linear system, the Maxwell's equations

$$
\begin{gathered}
\partial_{t} \boldsymbol{E}=c^{2} \nabla \times \boldsymbol{B}-\boldsymbol{J} / \epsilon_{0}, \\
\partial_{t} \boldsymbol{B}=-\nabla \times \boldsymbol{E},
\end{gathered}
$$

do not need be changed. We only need to seek a new linear system for $\boldsymbol{J}=\overleftrightarrow{\sigma} \cdot \boldsymbol{E}$. It is easy to find that after $J$-pole expansion, the relations between $\boldsymbol{J}$ and $\boldsymbol{E}$ has the following form:

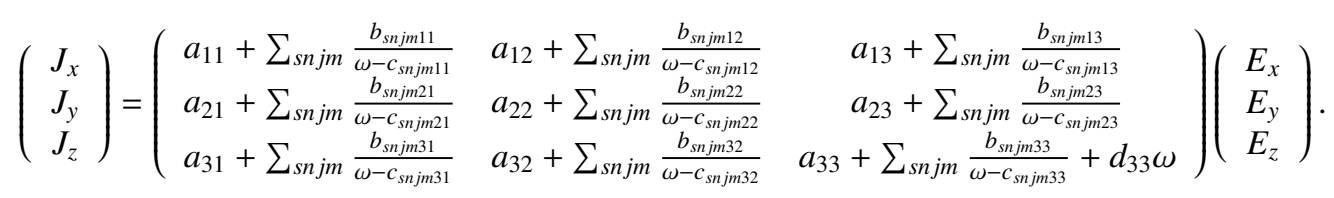

Fortunately, noting the relations in $Z$ function $\left(\sum_{j} b_{j}=-1, \sum_{j} b_{j} c_{j}=0\right.$ and $\left.\sum_{j} b_{j} c_{j}^{2}=-1 / 2\right)$ and in Bessel functions $\left[\sum_{n=-\infty}^{\infty} I_{n}(b)=e^{b}, \sum_{n=-\infty}^{\infty} n I_{n}(b)=0, \sum_{n=-\infty}^{\infty} n^{2} I_{n}(b)=b e^{b}\right.$, we find that $a_{i j}=0(i, j=1,2,3)$ and $d_{33}=0$. Eq. 97 can be changed further to

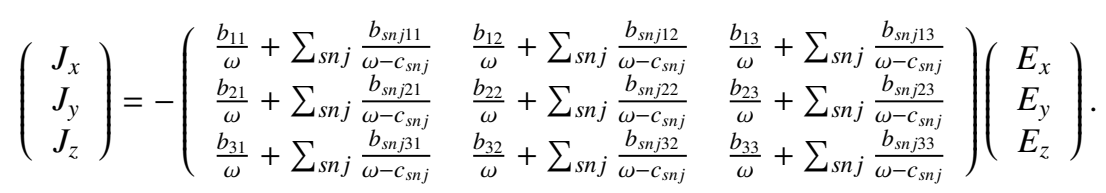

Combining Eqs. (8) and (10), the equivalent linear system for (6) can be obtained as

$$
\left\{\begin{array}{ccc}
\omega v_{s n j x} & = & c_{s n j} v_{s n j x}+b_{s n j 11} E_{x}+b_{s n j 12} E_{y}+b_{s n j 13} E_{z}, \\
\omega j_{x} & = & b_{11} E_{x}+b_{12} E_{y}+b_{13} E_{z}, \\
J_{x} & = & j_{x}+\sum_{s n j} v_{s n j x}, \\
\omega v_{s n j y} & = & c_{s n j} v_{s n j y}+b_{s n j 21} E_{x}+b_{s n j 22} E_{y}+b_{s n j 23} E_{z}, \\
\omega j_{y} & = & b_{21} E_{x}+b_{22} E_{y}+b_{23} E_{z}, \\
J_{y} & = & j_{y}+\sum_{s n j} v_{s n j y}, \\
\omega v_{s n j z} & = & c_{s n j} v_{s n j z}+b_{s n j 31} E_{x}+b_{s n j 32} E_{y}+b_{s n j 33} E_{z}, \\
\omega j_{z} & = & b_{31} E_{x}+b_{32} E_{y}+b_{33} E_{z}, \\
J_{z} & = & j_{z}+\sum_{s n j} v_{s n j z}, \\
\omega E_{x} & = & -c^{2} k_{z} B_{y}-J_{x} / \epsilon_{0}, \\
\omega E_{y} & = & c^{2} k_{z} B_{x}-c^{2} k_{x} B_{z}-J_{y} / \epsilon_{0}, \\
\omega E_{z} & = & c^{2} k_{x} B_{y}-J_{z} / \epsilon_{0}, \\
\omega B_{x} & = & k_{z} E_{y}, \\
\omega B_{y} & = & -k_{z} E_{x}+k_{x} E_{z}, \\
\omega B_{z} & = & -k_{z} E_{y},
\end{array}\right.
$$

which yields a sparse matrix eigenvalue problem. The elements of the eigenvector $\left(E_{x}, E_{y}, E_{z}, B_{x}, B_{y}, B_{z}\right)$ still represent the original electric and magnetic fields. Thus, the polarization of the solutions can also be obtained in a 
straightforward manner. The dimension of the matrix is $N N=3 \times(S N J+1)+6=3 \times[S \times(2 \times N+1) \times J+1]+6$. The coefficients are

$$
\left\{\begin{array}{ccc}
b_{s n j 11} & = & \omega_{p s}^{2} b_{j}\left(1-k_{z} b_{j 0} / c_{s n j}\right) n^{2} \Gamma_{n} / b_{s}, \\
b_{11} & = & \sum_{s n j} \omega_{p s}^{2} b_{j}\left(k_{z} b_{j 0} / c_{s n j}\right) n^{2} \Gamma_{n} / b_{s}, \\
b_{s n j 12} & = & \omega_{p s}^{2} b_{j}\left(1-k_{z} b_{j 0} / c_{s n j}\right) i n \Gamma_{n}^{\prime}, \\
b_{12}, & = & \sum_{s n j} \omega_{p s}^{2} b_{j}\left(k_{z} b_{j 0} / c_{s n j}\right) i n \Gamma_{n}^{\prime}, \\
b_{s n j 21}=-b_{s n j 12} & , & b_{21}=-b_{12}, \\
b_{s n j 22} & = & \omega_{p s}^{2} b_{j}\left(1-k_{z} b_{j 0} / c_{s n j}\right)\left(n^{2} \Gamma_{n} / b_{s}-2 b_{s} \Gamma_{n}^{\prime}\right), \\
b_{22} & = & \sum_{s n j} \omega_{p s}^{2} b_{j}\left(k_{z} b_{j 0} / c_{s n j}\right)\left(n^{2} \Gamma_{n} / b_{s}-2 b_{s} \Gamma_{n}^{\prime}\right), \\
b_{s n j 13} & = & \omega_{p s}^{2} b_{j}\left[c_{j} / \lambda_{T s}-n \omega_{c s} b_{j 0} /\left(c_{s n j} v_{t z s}\right)\right] \Gamma_{n} / b_{s}, \\
b_{13} & = & \sum_{s n j} \omega_{p s}^{2} b_{j}\left[n \omega_{c s} b_{j 0} /\left(c_{s n j} v_{t z s}\right)\right] \Gamma_{n} / b_{s}, \\
b_{s n j 31}=b_{s n j 13} & , & b_{31}=b_{13}, \\
b_{s n j 23} & = & -i \omega_{p s}^{2} b_{j}\left[c_{j} / \lambda_{T s}-n \omega_{c s} b_{j 0} /\left(c_{s n j} v_{t z s}\right)\right] \sqrt{\left(2 \lambda_{T s}\right) \Gamma_{n}^{\prime} b_{s},} \\
b_{23} & = & -i \sum_{s n j} \omega_{p s}^{2} b_{j}\left[n \omega_{c s} b_{j 0} /\left(c_{s n j} v_{t z s}\right)\right] \sqrt{\left(2 \lambda_{T s}\right) \Gamma_{n}^{\prime} b_{s},} \\
b_{s n j 32}=-b_{s n j 23} & , & b_{32}=-b_{23}, \\
b_{s n j 33} & = & \omega_{p s}^{2} b_{j}\left[\left(v_{s 0} / v_{t z s}+c_{j}\right) c_{j} / \lambda_{T s}-n \omega_{c s} b_{j 0}\left(1+n \omega_{c s} /\left(c_{s n j}\right) v_{t z s}^{2}\right) / k_{z}\right] 2 \lambda_{T s} \Gamma_{n}, \\
b_{33} & = & \sum_{s n j} \omega_{p s}^{2} b_{j}\left[n^{2} b_{j 0} /\left(c_{s n j} v_{t z s}^{2} k_{z}\right)\right] 2 \lambda_{T s} \Gamma_{n}, \\
c_{s n j} & = & k_{z} c_{j} v_{t z s}+k_{z} v_{s 0}-n \omega_{c s},
\end{array}\right.
$$

where $b_{j 0}=v_{s 0}+\left(1-1 / \lambda_{T s}\right) c_{j} v_{t z s}$.

If $a_{i j} \neq 0$, then the equivalent linear transformation is still straightforward. However, the eigenmatrix will not be sparse (the ES1D and ES3D eigenmatrices in Sec 2 are not sparse, see Appendix B for the sparse ones). If $d_{33} \neq 0$, then the equivalent linear transformation will be more complicated. For our purposes, we do not need to discuss these cases.

\section{Benchmarks and applications}

The PDRK code is developed based on the above method. We now benchmark this code and show some typical applications. Default parameters for the succeeding cases are $c^{2}=10^{4}, B_{0}=1, m_{e}=1, q_{e}=-1, \epsilon_{0}=1$.

\subsection{Benchmark with fluid solver PDRF}

First, we compare PDRK with the fluid solver PDRF[1]. Fig[5 shows the results at the cold limit with parallel propagation $\left(k=k_{z}\right.$ ). In PDRF, we set $T_{e}=T_{i}=0$; in PDRK, we set $T_{e}=T_{i}=0.01 \ll 1$. The real frequencies in PDRK $\left(\omega^{K}\right)$ and in PDRF $\left(\omega^{F}\right)$ are almost identical. However, the kinetic damping is not zero as in the fluid framework, especially the cyclotron damping for ions, which is apparent in Panel (b). This cyclotron damping is not predicted in the fluid theory.

Fig 6 shows the results for warm plasma with perpendicular propagation. We see that the fluid version results are close to the kinetic version results at small $k\left(k c / \omega_{c e}<2\right)$, but deviates at large $k$. This kinetic correction (Bernstein modes) from the harmonics of the cyclotron frequency is also not predicted in fluid theory.

A further test (Fig 12) of the electron Bernstein modes, which is quasi-electrostatic and makes use of the parameters in Fig 3 gives similar results between PDRK-EM3D and PDRK-ES3D. Thus, for this step, PDRK-EM3D works well.

\subsection{Parallel propagation kinetic modes}

The kinetic dispersion relation for parallel propagation modes [16, 18] is relatively simple to solve because the effects of the higher-order cyclotron harmonics are zero. One branch is the same as the ES1D dispersion relation Eq. (1). The other two branches are given by

$$
D(k, \omega)=1-\frac{k^{2} c^{2}}{\omega^{2}}+\sum_{s} \frac{\omega_{p s}^{2}}{\omega k v_{t s}} Z\left(\frac{\omega \pm \omega_{c s}}{k v_{t s}}\right)=0 .
$$



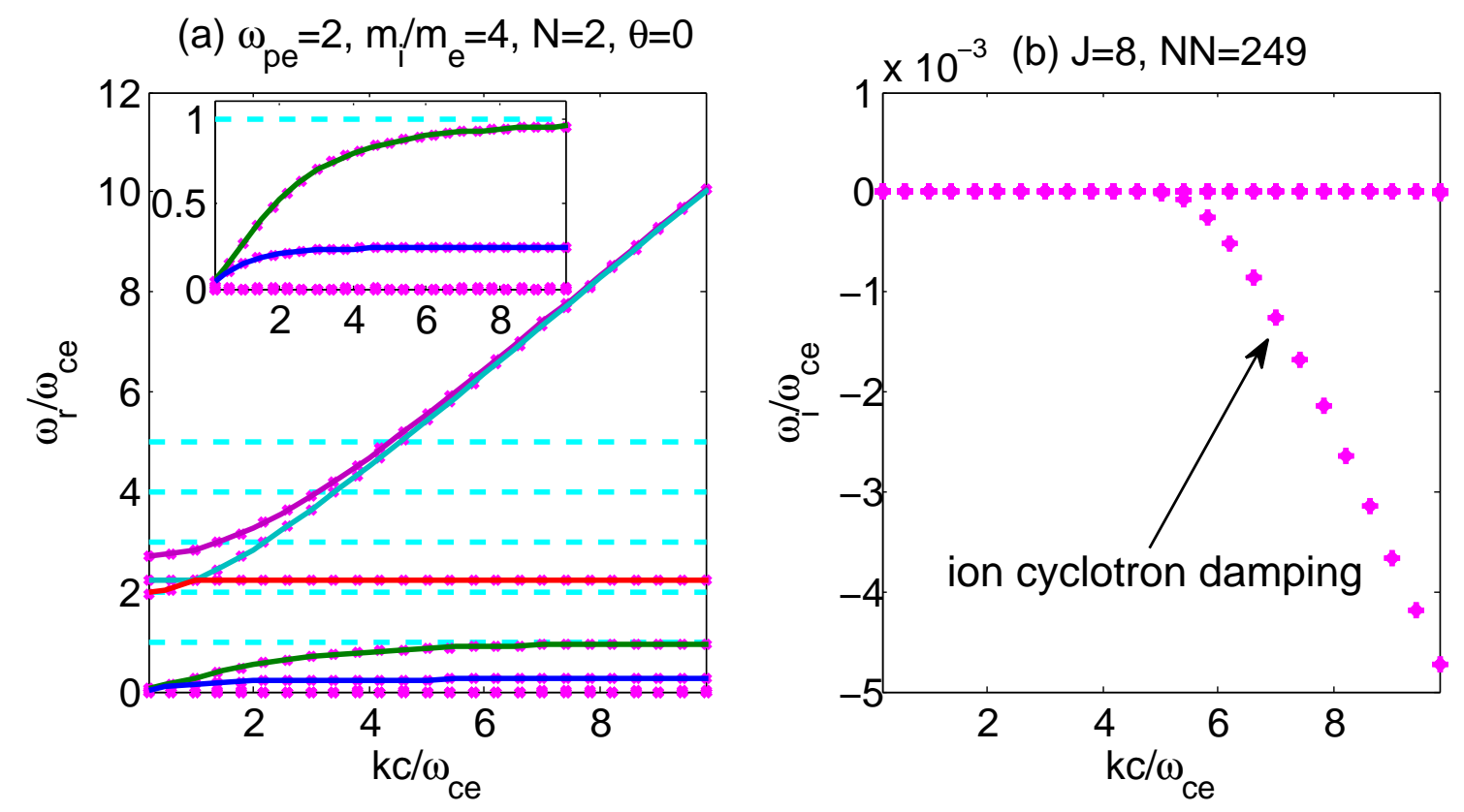

Figure 5: PDRK (dot) vs. PDRF (solid line), cold $\left(T_{e}=T_{i}=0.01\right)$, parallel propagation.

(a) $\omega_{p e}=2, m_{i} / m_{e}=4, N=3, \theta=90$

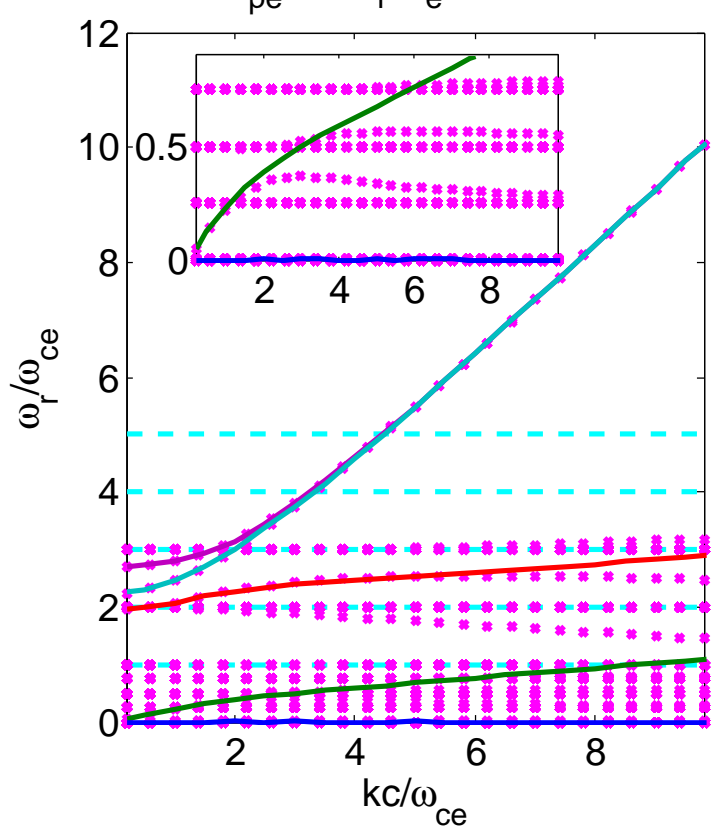

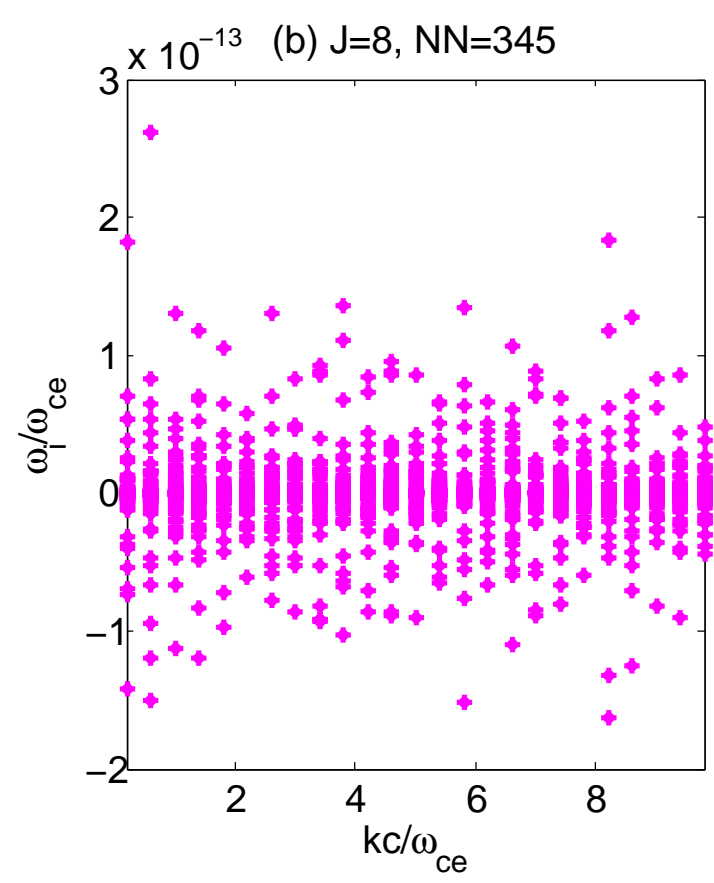

Figure 6: PDRK (dot) vs. PDRF (solid line), warm $\left(T_{e}=T_{i}=100\right.$ ), perpendicular propagation. The positive $\gamma \simeq 10^{-13}$ comes from numerical error of $J=8$. 
(a) $\omega_{p e}=3, m_{i} / m_{e}=4, N=2, \theta=0$

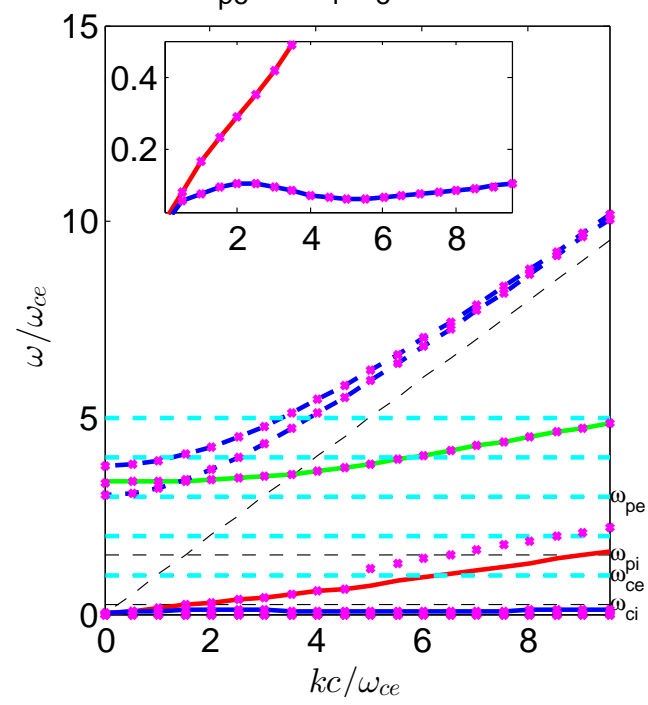

(b) $\mathrm{J}=8, \mathrm{NN}=249$

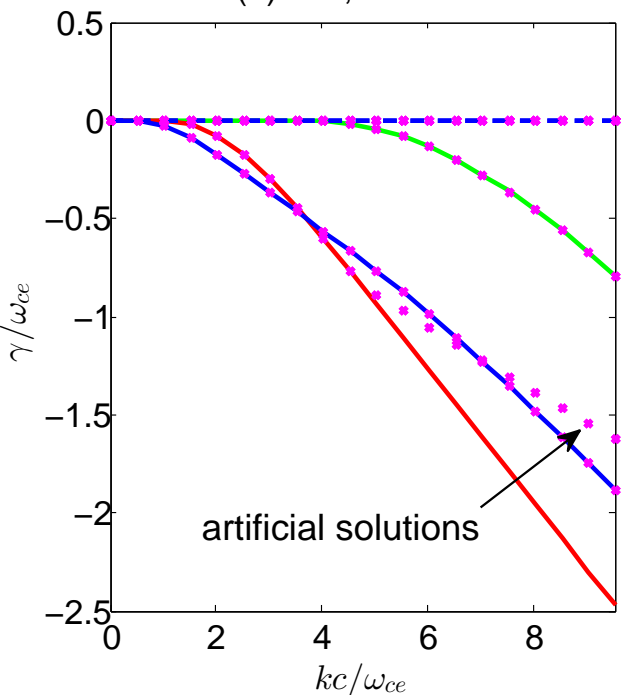

Figure 7: PDRK solutions (dot) vs. Z function solutions (solid and green dash lines), warm $\left(T_{e}=T_{i}=400\right.$ ), parallel propagation. Heavily damped (both real and artificial) solutions are not shown.

Eqs.(1) and (13) are solved by root finding with the original $Z$ function[19] and comparing with PDRK. A typical result is shown in Fig 7. We find a good agreement between the two methods. In addition, the ion and electron cyclotron damping and the Landau damping are clearly shown. However, too many extraneous solutions exist in the PDRK results. Most of the heavily damped solutions are not shown in the figure. The solutions represented by the red solid line $\left(\omega^{R}\right)$ in the figure should be real solutions. At large $k$ (e.g., $k c / \omega_{c e}>7$, where PDRK solutions still agree with $\omega^{R}$ but not shown), the damping rate of several artificial solutions are smaller than $\omega^{R}$, which makes it difficult to separate the real and artificial solutions directly.

To this step, PDRK-EM3D works well for $T_{s \|}=T_{s \perp}$ and $v_{s 0}=0$. For the heavily damped solutions, keeping all the interesting solutions while removing the artificial solutions is usually not easy. Besides the heavily damped solutions, the artificial solutions roughly satisfy $\omega_{r}-n \Omega_{c} \propto k_{\|}$and $\gamma \propto k_{\|}$(come from the poles $\zeta-c_{j} \rightarrow 0$ of $J$-pole expansion). Therefore, this process can also be used to remove some of the artificial solutions. Several of the ES3D artificial solutions in Fig 8 are removed based on this property.

When a sparse matrix is not used, the computation time is around $O\left(N N^{\alpha}\right)$ with $2<\alpha<3$ and the memory required is around $O\left(N N^{2}\right)$. A typical personal computer with $4 \mathrm{~GB}$ memory can calculate $N N$ up to $7000(N N=7000, S=2$, $J=8$, give $N \simeq 60$ ) in minutes. Thus, for modes with frequency $\omega<60 \Omega_{c i}$, all the solutions in the system can be obtained easily. When a sparse matrix is used, $N N$ can reach up to $10^{6}$. Thus $N$ can be up to $10^{4}$. The standard sparse matrix algorithm can solve one or several solutions around the initial guess.

\subsection{Landau damping of lower hybrid wave}

Now, we benchmark the Landau damping of lower hybrid wave (LHW) using a real mass ratio $m_{i} / m_{e}=1836$, where large $N$ should be used to make the solutions convergent. For the electrostatic case, with $k^{2} \rho_{e}^{2} \ll 1, \omega_{c i} \ll \omega \ll$ $\omega_{p e}$ and $k_{\|} / k \ll 1$, the analytical solution $\omega=\left(\omega_{r}, \gamma\right)$ for LHW can be found in Ref.[20]. We use the same parameters $\left(\omega_{p e}=\omega_{c e}, k_{\|} / k_{\perp}=0.066, T_{e}=T_{i}\right)$ as in the Fig.1 of Ref.[20] for the benchmark because this has also been verified by first-principle PIC simulations in that paper. The results are shown in Fig 8 , where the electrostatic assumption works well for large $k$. For small $k\left(k_{\perp} \rho_{c e}<0.04\right)$, the electromagnetic effects should be included, which is consistent with the results on fluid frequency and polarization in a previous study[1].

Note that several limits for the parameters have been used to obtain the analytical solution. Similar limits have also been used for warm EM LHW (see e.g., [8]). Therefore, it is not surprising that the analytical solution does not 

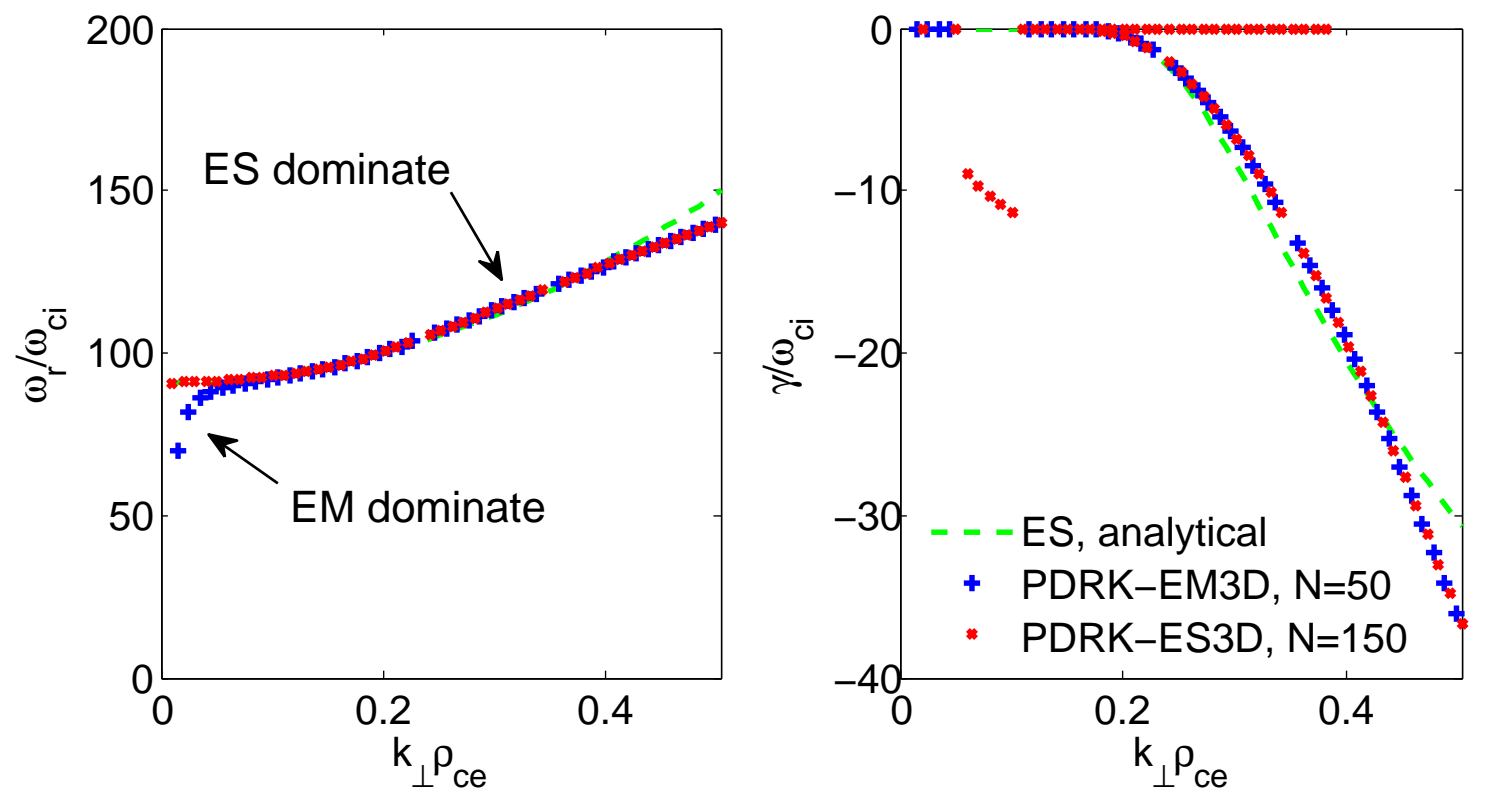

Figure 8: Landau damping of lower hybrid wave. Solutions from PDRK-ES3D (red, $N=150$ ), PDRK-EM3D (blue, $N=50$ ), and the analytical solution (dash green line) in Ref.[20]. It took about $1 \mathrm{CPU}$ hour to compute the data in this figure.

hold for large $k\left(k_{\perp} \rho_{c e}>0.4\right)$ in the figure. For fusion (e.g., [21]) or space studies, the approximate analytical solution is not always valid. Thus, PDRK can serve as a numerical tool for a wider range of parameters.

For this step, we have shown that PDRK-EM3D works well also for $N \geq 50$ by using a sparse matrix, although an initial guess is required and the computational time is longer.

\subsection{Firehose and mirror modes}

Firehose and mirror modes are typical unstable modes driven by pressure anisotropic $T_{\|} \neq T_{\perp}$. For cold electrons, the approximate analytical kinetic dispersion relations for the firehose mode is $\omega^{2}=\omega_{A}^{2}\left[\frac{b_{i}}{1-\Gamma_{0}\left(b_{i}\right)}+\frac{\beta_{i \perp}-\beta_{i \|}}{2}\right]$. For the mirror mode, it is $\zeta_{i} Z\left(\zeta_{i}\right)=\frac{\eta_{i}}{\beta_{i \perp} \Gamma_{1}\left(b_{i}\right)}-\left(1-\eta_{i}\right)$, with $\eta_{i}=\beta_{i \|} / \beta_{i \perp}$.

A typical result is shown in Fig 9 , where $\omega_{p e} / \omega_{c e}=2, m_{i} / m_{e}=100, \omega_{A}=k_{\|} v_{A}=0.01 \omega_{c i}$ and $\beta_{e}=0.08$. The PDRK solutions agree with the analytical solutions for both the firehose and mirror modes. The small deviation is not surprising because the analytical solutions are not accurate.

\subsection{Whistler beam mode}

The beam $v_{s 0} \neq 0$ can also drive instabilities. We benchmark the whistler beam mode here. The parameters are similar to Fig.8.8 of Ref.[5], with $s=b, c, i, m_{i} / m_{e}=1836, n_{i}=1.0 e 4, n_{b}=0.1 n_{i}, n_{c}=0.9 n_{i}, T_{c}=T_{i}=T_{b} / 10=$ 0.5556 and $v_{b 0}=-9 v_{c 0}=2.108$, which yield $\omega_{p e}=100 \omega_{c e}, \beta_{c}=1.0$ and $v_{b 0}=2.0 v_{t c}$. The $\omega$ and $\gamma$ vs. $\left(k_{z}, k_{x}\right)$ results are shown in Fig 10 . The most unstable mode is the parallel propagation mode $\left(k=k_{\|}\right)$, which is consistent with Gary's conclusion[5].

\subsection{New anomalous Doppler shift}

With PDRK, it was the first time that we can see a complete picture of the waves and instabilities in a kinetic system. New modes which are unknown in previous studies, may now be found. Several examples of new modes have been found by PDRK. We show one of them here, namely, a new anomalous Doppler effect.

The Lorentz Doppler shift for relativistic cold fluid plasma has been verified by PDRF[1]. Here, we are interested in the Doppler asymmetry of the electron and ion beams in kinetic non-relativistic plasmas. The parameters $\left(\omega_{p e}=\right.$ $100 \omega_{c e}, m_{i}=1836 m_{e}, T_{i}=T_{e}=0.01, \theta=1.5528=88.97^{\circ}$ and $\left.v_{d}=0.99\right)$ are taken similar to those in Fig. 1 

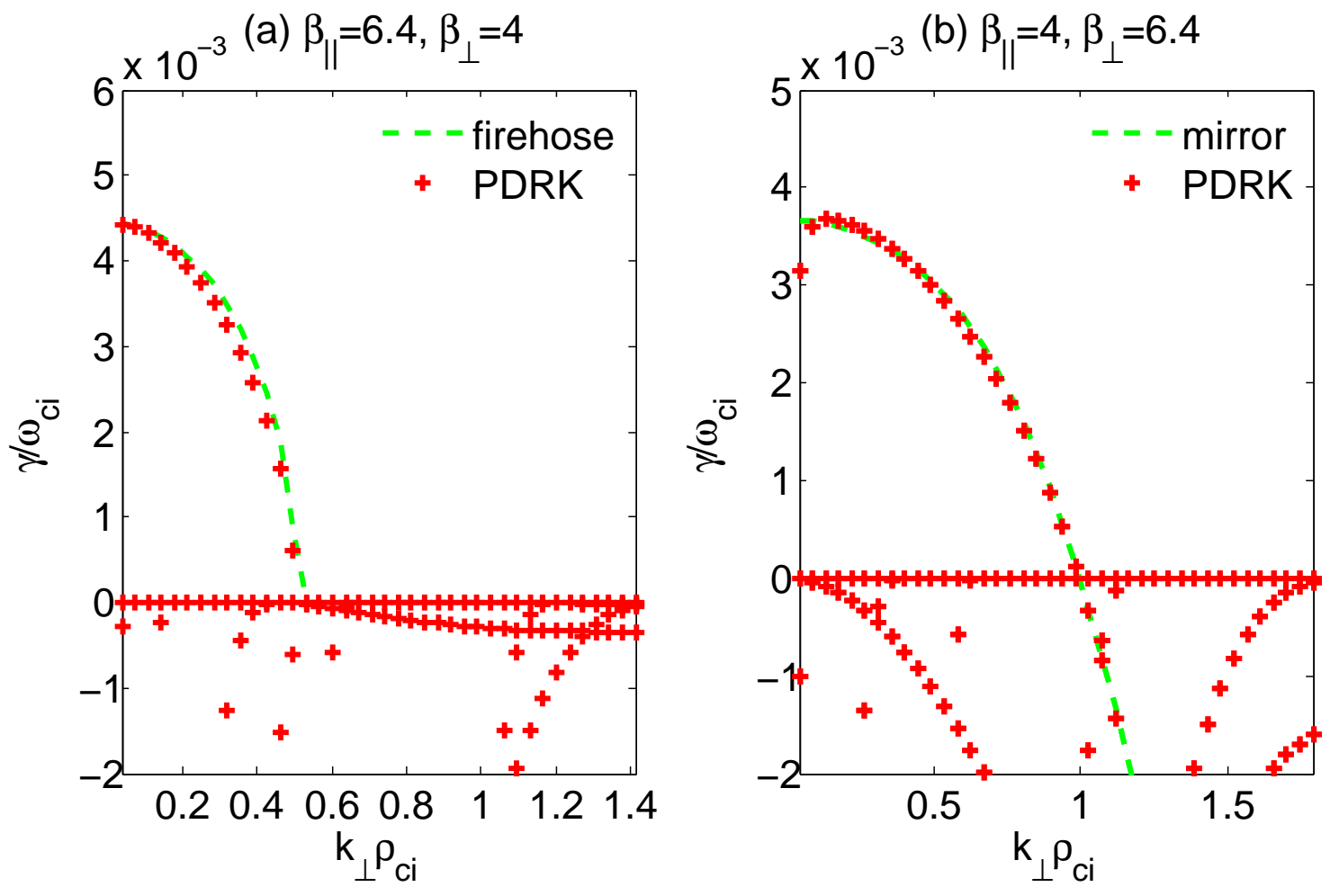

Figure 9: Growth rates for the firehose and mirror modes vs. $k_{\perp} \rho_{c i}$. The dashed green lines are analytical solutions.
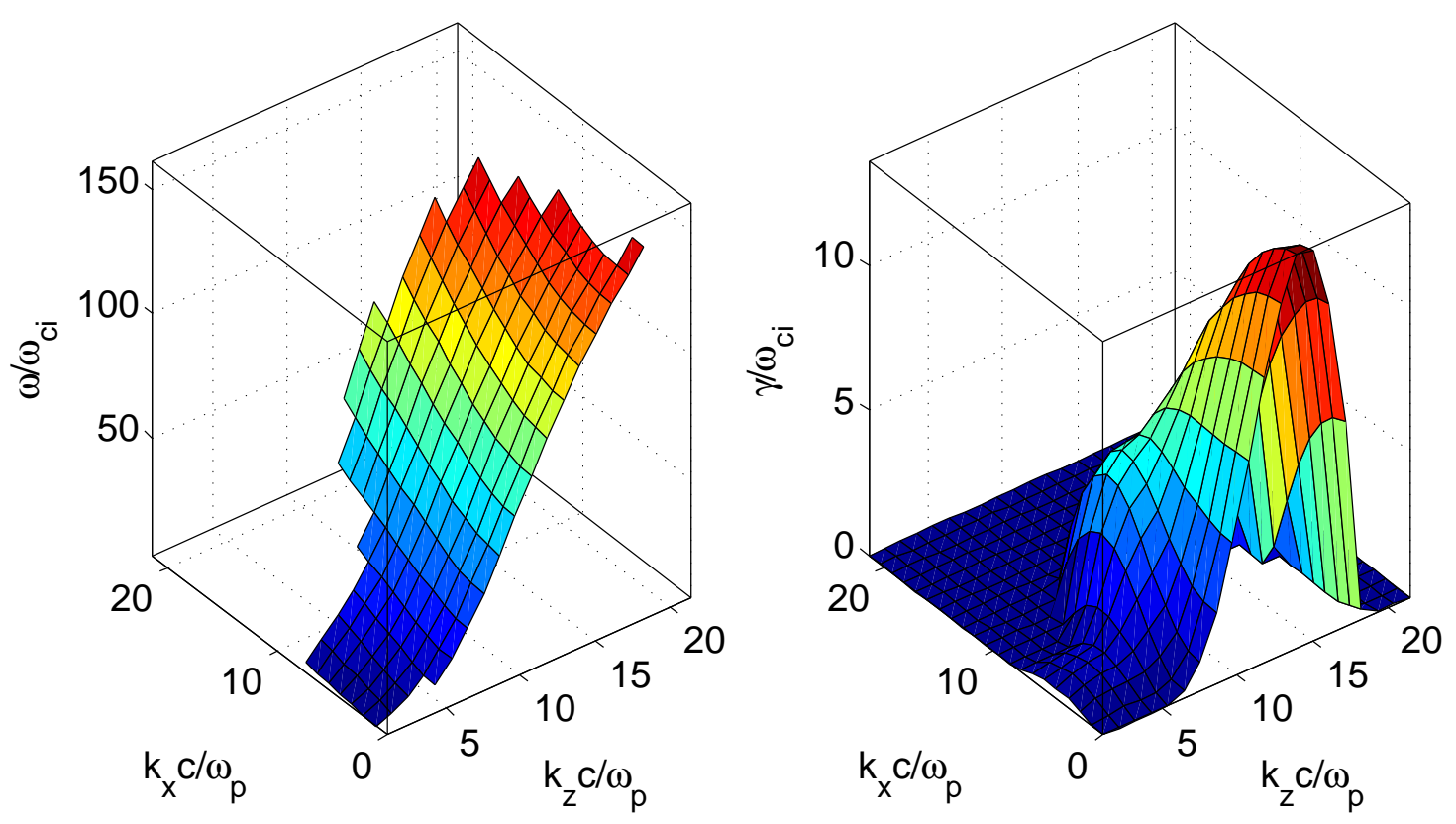

Figure 10: Electromagnetic whistler beam instability. The real frequency $\omega$ is only shown for unstable $(\gamma>0)$ solutions. The parallel propagation $\left(k=k_{\|}\right)$results are similar to Fig.8.8 of Ref.[5]. $N=3$ is used for this calculation. 

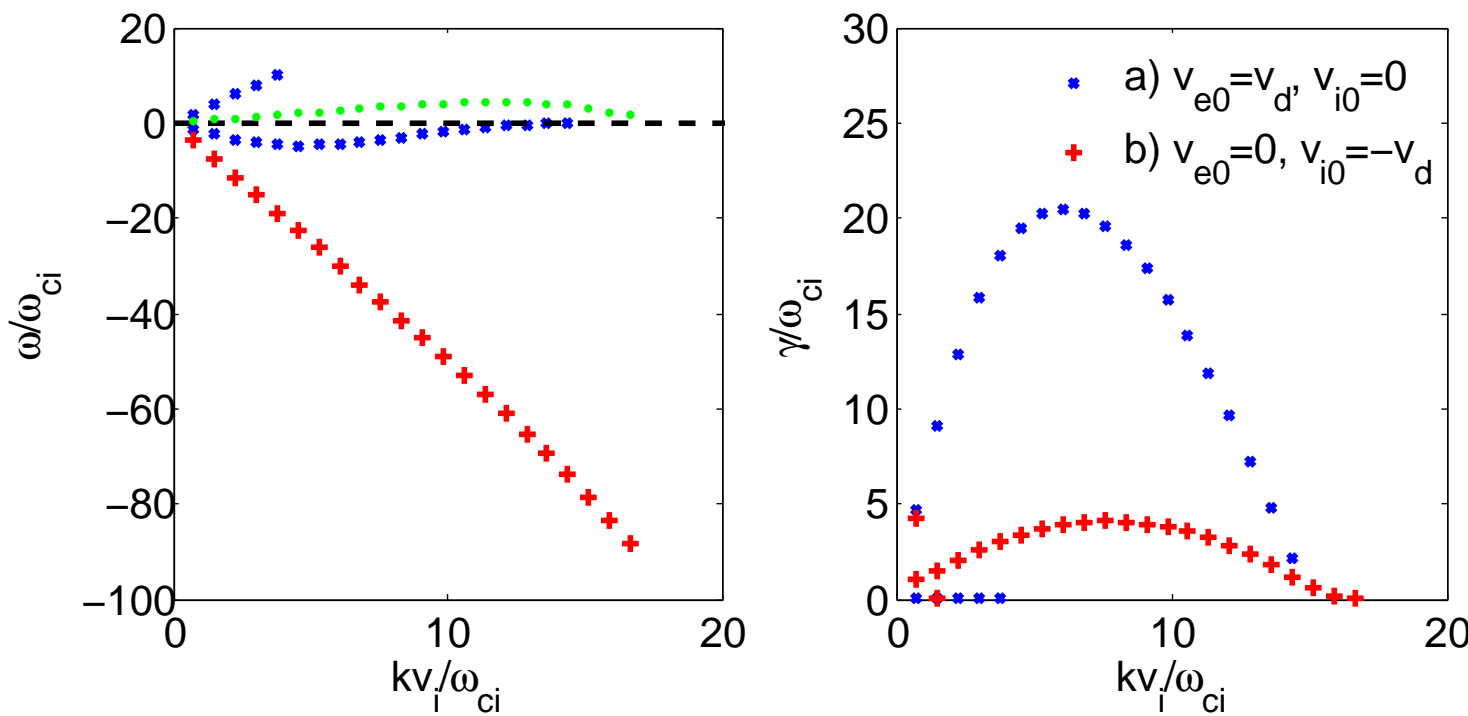

Figure 11: New modes found by PDRK-EM3D: the Doppler asymmetry of electron and ion beams in kinetic non-relativistic plasmas. Blue ' $X$ ' for a); red '+' for b); green dot is $\omega=\omega^{b}-k_{\|} v_{d}$.

of Ref.[22] for instability of the lower hybrid-like waves driven by parallel current. The current is taken by electron beam in Ref.[22]. We also consider an ion beam and solve the dispersion relations for the following two cases: (a) $v_{e 0}=v_{d}, v_{i 0}=0$; (b) $v_{e 0}=0, v_{i 0}=-v_{d}$. Here, the thermal velocity $v_{t s}$ and drift velocity $v_{d}$ are all non-relativistic, i.e., $v_{t s}, v_{d}<0.01 c \ll c$. If the system is Galilean invariant, then the solution $\omega^{a}$ for (a) and $\omega^{b}$ for (b) should satisfy $\omega^{a}=\omega^{b}-k_{\|} v_{d}$. The foregoing also means that the growth rate will not change $\left(\gamma^{a}=\gamma^{b}\right)$ for the same $k$.

Evidently, the ES dispersion relations (1) and (5) are Galilean types, i.e., $\omega^{a}=\omega^{b}-k_{\|} v_{d}$. In common understanding, the EM3D dispersion relation (6) should also be Galilean when $v_{t s}, v_{d} \ll c$. However, as the results show in Fig 11 , we find that the Galilean Doppler effect is not present for both real frequency and growth rate, i.e., $\omega^{a} \neq \omega^{b}-k_{\|} v_{d}$ and $\gamma^{a} \neq \gamma^{b}$. Detailed discussion of the physics behind this interesting result is not within the scope of the present work and may be explored further. The purpose of the result shown here is to demonstrate that PDRK can be useful and effective in revealing new modes.

\subsection{Dispersion surface}

The 2D structure of $\omega$ vs. $\left(k_{x}, k_{z}\right)$ (dispersion surface[23]) is shown in Fig 12 for electron Bernstein wave (EBW). This type of figure is helpful in displaying the fine structure of the dispersion relations in $\left(k_{\perp}, k_{\|}\right)$space and in revealing the relations among different modes. It is clearly shown in Panel (b) that the solutions are separated by cyclotron frequencies, i.e., the solution $n \omega_{c}<\omega<(n+1) \omega_{c}(n=0,1,2, \ldots)$ exists for any $k$. In Fig 12 , we only keep $N=10$, and both real and artificial solutions are shown. To see the fine structure of the real solutions more clearly, further processing is required to remove the artificial solutions, which is the main disadvantage of the present version of PDRK.

\subsection{Others}

In the above benchmarks, no apparent numerical problems are found. However, this does not mean that we can apply PDRK for all cases because only approximations of $Z$ function are used. In WHAMP[2], the $Z$ function is also approximated but $J$-pole expansion is used. A further approximation is needed for the Bessel function summation. Thus, in principle, PDRK-EM3D will give more accurate results than WHAMP. Similar issues regarding the validity of Padé approximation for $Z$ is discussed in detail in the WHAMP report[2]. Based on our results, the error for $J=8$ is less than $10^{-4}$, which may bring some artificial growing modes. If the same solution also exists for other $J$ (e.g., $J=4,12$ ), it is more likely to be a real solution. Otherwise, care should be exercised in treating this solution. We 

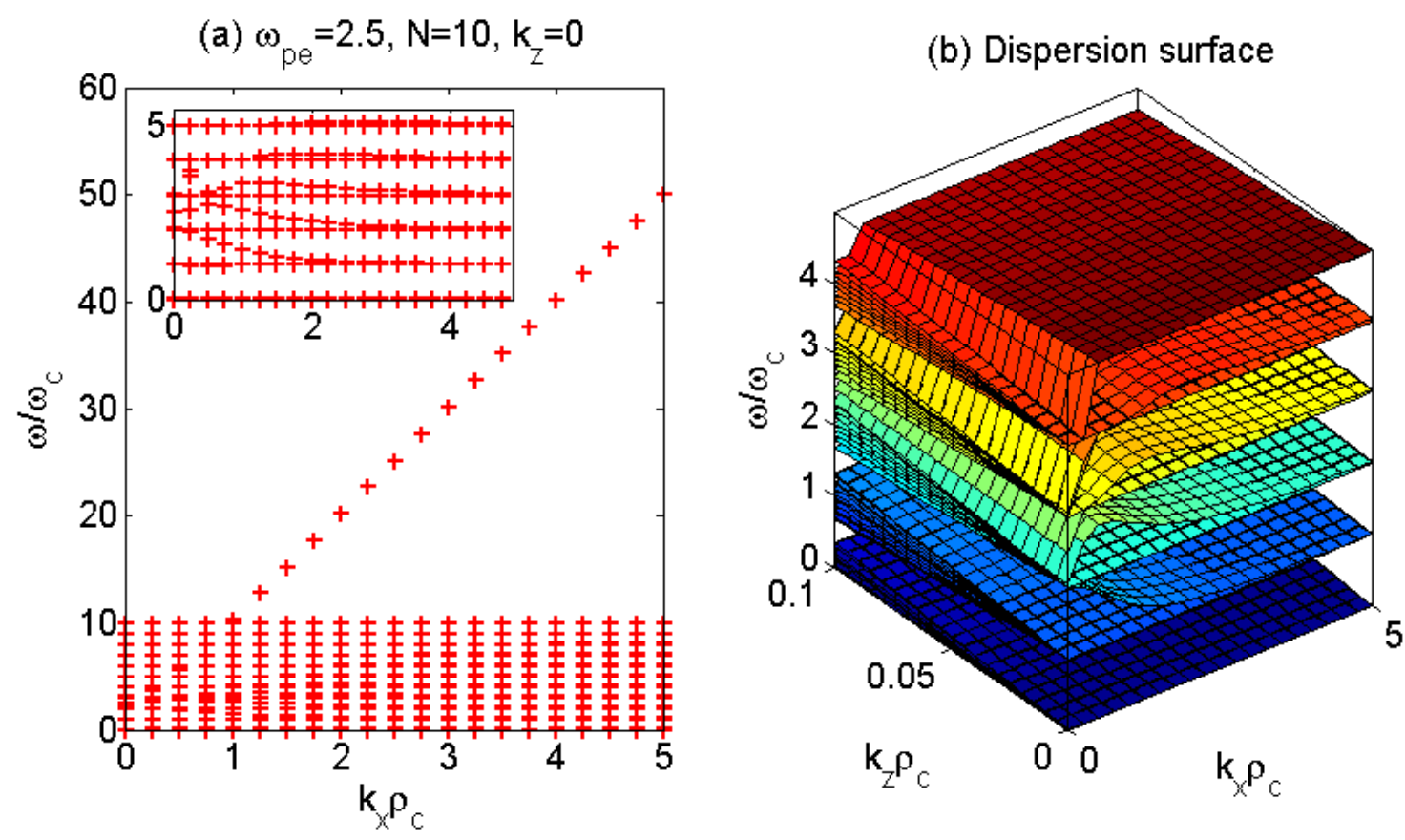

Figure 12: Dispersion surface (b) from PDRK-EM3D, using the EBW parameters in Fig 3 and $c^{2}=10^{2}$. The $\omega$ vs. $k_{\perp}$ (a) result is close to the ES3D result in Fig 3 which confirms that EBW is (quasi-) electrostatic.

can distinguish real and artificial solutions by using different $J$. The artificial solutions change when $J$ changes. By contrast, the real solutions do not change that much.

\section{Summary and discussion}

A general kinetic plasma dispersion relation solver, PDRK (three versions are included at present: ES1D, ES3D, EM3D), is developed, where the equilibrium distribution function is assumed to be drift bi-Maxwellian. For other non-Maxwellian distribution functions, the $J$-pole expansion Appendix A of the corresponding new $Z$ functions [14] should be obtained first. Note that the relativistic effect (e.g., [24-26]) is not included in the present study as this would make the solution more complicated. However, in principle, it can also be treated using Padé approximation[25]. Although PDRK is more accurate than PDRF, the latter is still advantageous in some cases because it can handle more configurations, such as relativistic systems, local non-uniform systems, and systems where collisions are considered. In addition, it does not produce artificial solutions. For practical applications, one can use PDRF to obtain rough solutions, and then use these to provide initial guesses for PDRK or use them for assistance in removing the artificial solutions in PDRK. Besides the multi-fluid model, PDRK also provides a tool to check the validity of other reduced models, such as Darwin[19] and gyro-kinetic[9, 27] models.

For systems with small $N$ (e.g., $N<60$ for two species) or unstable modes, PDRK works excellently and is applicable to most cases used. For large $N$ (e.g., $N>60$ ), especially in studying the effect of $n \Omega_{c}$ to the modes (e.g., LHW), the performance of PDRK is limited mainly by the computational time and memory. However, this concern may be remedied by using sparse matrices. Further optimization is possible. For example, we do not need to treat $N$ equally for each species, e.g., for LHW, we can use large $N_{i}$ but small $N_{e}$. The main disadvantage of PDRK is that the artificial solutions originate from the poor approximation for strongly damped modes.

Compared with conventional solvers, the PDRK solver is fast and can give all solutions. Therefore, no important solutions are missed. It is also free from convergence problems. Hence, this solver can find wide applications in space, astrophysical, laser, and laboratory plasma studies. 


\section{Acknowledgements}

The work is supported by the National Magnetic Confinement Fusion Science Program under Grant No. 2011GB105001 and 2013GB111000, China NSFC under Grant No. 91130031, the Recruitment Program of Global Youth Experts.

\section{Appendix A. Arbitrary $J$-pole expansion}

The $J$-pole expansion coefficients $b_{j}$ and $c_{j}$ are provided only for small $J$ in literature. Here, based on the study of Ronnmark[2], we develop a scheme to calculate the numerical coefficients for any $J$. This is possible because we do not need the analytical expressions. The $J$-pole expansion is

$$
Z(\zeta) \simeq Z_{A}^{J}(\zeta)=\frac{\sum_{k=0}^{J-1} p_{k} \zeta^{k}}{q_{0}+\sum_{k=1}^{J} q_{k} \zeta^{k}},
$$

with $q_{0}=1$, should be matched with the following two-side approximation

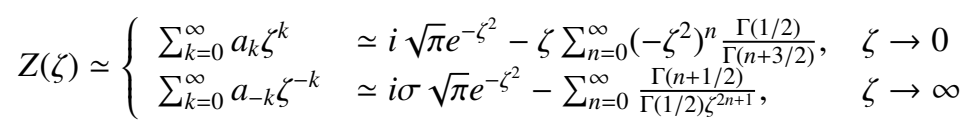

where

$$
\sigma= \begin{cases}0, & \operatorname{IM}(\zeta)>0 \\ 1, & \operatorname{IM}(\zeta)=0 \\ 2, & \operatorname{IM}(\zeta)<0\end{cases}
$$

and $\Gamma$ is Euler's Gamma function. A further expansion is $e^{-\zeta^{2}}=\sum_{n=0}^{\infty} \frac{\zeta^{2 n}}{n !}$. However, $i \sigma \sqrt{\pi} e^{-\zeta^{2}}$ is omitted, which does not match well for the range $y<\sqrt{\pi} x^{2} e^{-x^{2}}$ when $x \gg 1$. The system of equations to be solved are

$$
\begin{gathered}
p_{j}=\sum_{k=0}^{j} a_{k} q_{j-k}, 1 \leq j \leq I \\
p_{L-j}=\sum_{k=0}^{j} a_{-k} q_{L+k-j}, 1 \leq j \leq K
\end{gathered}
$$

where $I+K=2 J$, and $p_{j}=0$ for $j>J-1$ and $j<0$, and $q_{j}=0$ for $j>J$ and $j<0$. Thus $2 J$ equations determine $2 J$ coefficients $p_{j}$ and $q_{j}$ in A.1. The derivation of (A.4) is similar to that of Eqs.(III-5) and (III-7) in Ronnmark[2]. Eqs.(A.4) are solved using matrix inversion. The 'residue()' function in MATLAB is used to calculate $b_{j}$ and $c_{j}$ in (2) from A.1. The results for $J=12$ using $I=16$ equations of A.4a and $K=8$ equations of A.4b are given in Table 1.

Usually, a large $J$ gives better approximations. However, this is not always the case. Test should be made before using them. Moreover, the truncated error when using double precision data can accumulate to $10^{-11}$.

Calculating the $J$-pole expansions for other equilibrium distribution functions [14] is also straightforward. We merely replace the coefficients $a_{k}$ and $a_{-k}$ in A.2.

\section{Appendix B. Equivalent sparse matrix for ES1D system}

As mentioned, the equivalent matrix from Eq.(4) for ES1D system is not sparse. An equivalent sparse matrix for ES1D system can be constructed as following:

$$
\begin{gathered}
\omega n_{s j}=c_{s j} n_{s j}+b_{s j} E, \\
\omega E=-\sum_{s j} c_{s j} n_{s j}-\sum_{s j} b_{s j} E .
\end{gathered}
$$

This is similar by changing the ES1D Vlasov-Poisson system to the ES1D Vlasov-Ampere system[15]. Eq.[B.1b] can be further simplified to be $\omega E=-\sum_{s j} c_{s j} n_{s j}$, because $\sum_{s j} b_{s j}=0$. The ES3D matrix in Sec 2.2 can be changed to sparse matrix in a similar manner. 


\section{Appendix C. PDRK User Manual}

The structure of PDRK is similar to that of PDRF, i.e., it contains two files: the main program "pdrk.m" and the input data file "pdrk.in". The input file has the following structure

$\begin{array}{llllll}\text { qs } & \mathrm{ms} & \mathrm{ns} & \mathrm{Tzs} & \mathrm{Tps} & \mathrm{vs0} \\ -1.0 & 1.0 & 4.0 & 1.0 & 1.0 & 0.0 \\ 1.0 & 4.0 & 4.0 & 1.0 & 1.0 & 0.0\end{array}$

More species can be added directly to new lines. Implementing "pdrk.m" in other languages (e.g., Fortran, C/C++, Python) is also straightforward.

\section{References}

[1] H. S. Xie, Computer Physics Communications, 185 (2014) 670.

[2] K. Ronnmark, KGI Report No. 179, Sweden, 1982.

[3] K. Ronnmark, Plasma Physics, 25 (1983) 699.

[4] D. Verscharen, S. Bourouaine, B. D. G. Chandran and B. A. Maruca, The Astrophysical Journal, 773 (2013) 8

[5] S. P. Gary, Theory of Space Plasma Microinstabilities, Cambridge, 1993.

[6] S. P. Gary, K. Liu and D. Winske, Phys. Plasmas 18 (2011) 082902.

[7] A. J. Willes and I. H. Cairns, Phys. Plasmas 7 (2000) 3167.

[8] A. L. Verdon, I. H. Cairns, D. B. Melrose and P. A. Robinson, Phys. Plasmas 16 (2009) 052105.

[9] Y. Lin, X. Y. Wang, Z. Lin and L. Chen, Plasma Phys. Control. Fusion 47 (2005) 657.

[10] P. Martin, G. Donoso and J. Zamudio-Cristi, J. Math. Phys. 21 (1980) 280.

[11] C. Cereceda and J. Puerta, Phys. Scr. 2000 (2000) 206.

[12] A. Tjulin, A. I. Eriksson, and M. J. Andre, Plasma Physics 64 (2000) 287

[13] P. A. Robinson and D. L. Newman, J. Plasma Physics 40 (1988) 553.

[14] H. S. Xie, Phys. Plasmas 20 (2013) 092125.

[15] H. S. Xie, Phys. Plasmas 20 (2013) 112108.

[16] D. A. Gurnett and A. Bhattacharjee, Introduction to plasma physics: with space and laboratory applications, Cambridge, 2005.

[17] S. J. Gitomer, D. W. Forslund and L. Rudsinski, Physics of Fluids, 15 (1972) 1570.

[18] T. Stix, Waves in Plasmas, AIP Press, 1992.

[19] H. S. Xie, J. Zhu and Z. W. Ma, Phys. Scr. 89 (2014) 105602.

[20] L. Qi, X. Y. Wang and Y. Lin, Phys. Plasmas 20 (2013) 062107.

[21] J. Bao, Z. Lin, A. Kuley and Z. X. Lu, Plasma Phys. Control. Fusion 56 (2014) 095020.

[22] A. L. Verdon, I. H. Cairns, D. B. Melrose and P. A. Robinson, Phys. Plasmas 18 (2011) 082103.

[23] M. André, J. Plasma Physics, 33 (1985) 1.

[24] A. Bret, L. Gremillet and D. Bénisti, Phys. Rev. E 81 (2010) 036402.

[25] B. Hao, W. J. Ding, Z. M. Sheng, C. Ren, X. Kong, J. Mu and J. Zhang, Phys. Plasmas 19 (2012) 072709.

[26] I. V. Timofeev and V. V. Annenkov, Phys. Plasmas 20 (2013) 092123.

[27] G. G. Howes, S. C. Cowley, W. Dorland, G. W. Hammett, E. Quataert and A. A. Schekochihin, The Astrophysical Journal 651 (2006) 590. 